\title{
New insights on the clinical variability of FKBP10 mutations
}

\author{
Osama H. Essawi ${ }^{\mathrm{a}, \mathrm{b}, *}$, Piyanoot Tapaneeyaphan ${ }^{\mathrm{b}}$, Sofie Symoens ${ }^{\mathrm{b}}$, Charlotte Gistelinck $\mathrm{C}^{\mathrm{c}}$, \\ Fransiska Malfait $^{\mathrm{b}}$, David R. Eyre ${ }^{\mathrm{c}}$, Tamer Essawi ${ }^{\mathrm{a}}$, Bert Callewaert ${ }^{\mathrm{b}}$, Paul J. Coucke ${ }^{\mathrm{b}}$ \\ ${ }^{a}$ Department Master Program in Clinical Laboratory Science, Birzeit University, Palestine \\ ${ }^{\mathrm{b}}$ Center for Medical Genetics, Ghent University, Ghent, Belgium \\ ${ }^{\mathrm{c}}$ Department of Orthopaedics and Sports Medicine, University of Washington, Seattle, WA, USA
}

\section{A R T I C L E I N F O}

\section{Keywords:}

FKBP10 gene

FKBP65 protein

Osteogenesis imperfecta (OI)

Bruck syndrome (BS)

Kuskokwim syndrome (KS)

Arthrogryposes

\begin{abstract}
A B S T R A C T
To date 45 autosomal recessive disease-causing variants are reported in the FKBP10 gene. Those variant were found to be associated with Osteogenesis Imperfecta (OI) for which the hallmark phenotype is bone fractuers or Bruck Syndrome (BS) where bone fractures are accompanied with contractures. In addition, a specific homozygous FKBP10 mutation (p.Tyr293del) has been described in Yup'ik Inuit population to cause Kuskokwim syndrome (KS) in which contractures without fractures are observed. Here we present an extended Palestinian family with 10 affected individuals harboring a novel homozygous splice site mutation, c.391 $+4 \mathrm{~A}>\mathrm{T}$ in intron 2 of the FKBP10 gene, in which the three above mentioned syndromes segregate as a result of skipping of exon 2 and absence of the FKBP65 protein. At the biochemical level, Hydroxylysyl pyridinoline (HP)/lysyl pyridinoline (LP) values were inversely correlated with OI phenotypes, a trend we could confirm in our patients. Our findings illustrate that single familial FKBP10 mutations can result in a phenotypic spectrum, ranging from fractures without contractures, to fractures and contractures and even to only contractures. This broad intrafamilial clinical variability within one single family is a new finding in the field of bone fragility.
\end{abstract}

\section{Introduction}

Osteogenesis imperfecta (OI) is a heterogeneous heritable connective tissue disorder, also known as "brittle bone disease", that is characterized by bone fragility, deformity and significant growth deficiency. This skeletal dysplasia may manifest other features including blue sclerae, hearing loss and dentinogenesis imperfecta. The underlying defects result in deficiency or structural abnormality of type I collagen, the main protein of tendon, skin and bone. The most frequent forms of OI are autosomal dominantly inherited and caused by mutations in COL1A1 (OMIM 120150) or COL1A2 (OMIM 120160) encoding type I collagen monomers. The spectrum of associated phenotypes vary from prenatal death to a very low fracture frequency (Sillence et al., 1979; Bonafe et al., 2015).

Since 2006 the molecular basis of several rare recessive forms of OI was identified which involve abnormalities in procollagen posttranslational modification, collagen folding and crosslinking, bone formation and mineralization (Sup. Table 1) (Forlino and Marini, 2016).

Defects in FKBP10 (OMIM 607063) which encodes FKBP65 protein, cause autosomal recessive OI (type $\mathrm{XI}$ ). This chaperone molecule localizes in the endoplasmic reticulum and belongs to the peptidylprolyl cis-trans isomerase (PPIase) family (Alanay et al., 2010; Ishikawa et al., 2008). FKBP65 protein is believed to play an important role in the hydroxylation of collagen telopeptide lysines, the essential process for generating collagen cross links that provide fibril tensile strength and stability. Also, FKBP65 deficiency was associated with reduced collagen deposition into the extracellular matrix (Barnes et al., 2012).

FKBP65 deficiency was initially reported in patients with severe OI, progressive scoliosis, and deforming long bone fractures in the absence of blue sclerae, hearing loss, and dentinogenesis imperfecta (Alanay et al., 2010). Subsequently, several reports associated FKBP65 with Bruck syndrome 1 (BRKS1, OMIM 259450), an autosomal recessive disorder characterized by congenital joint contractures, fragile bones, severe limb deformities, and progressive scoliosis (Shaheen et al., 2010, 2011; Kelley et al., 2011). A second cause of Bruck syndrome (BRSK2, OMIM 609220) results from defects in PLOD2 (OMIM 601865), encoding lysyl hydroxylase 2 (LH2), the enzyme responsible for collagen telopeptide lysine hydroxylation (Puig-Hervas et al., 2012).

Further studies report on FKBP10 pathogenic variants presenting with or without contractures within a same family, indicating that BRSK1 and OI are allelic disorders with variable occurrence of

\footnotetext{
${ }^{*}$ Corresponding author. Center of Medical Genetics Ghent University Hospital Medical Research Building 1 (MRB 1) C. Heymanslaan 10, 9000, Ghent, Belgium.

E-mail address: osama.essawi@Ugent.be (O.H. Essawi).
} 
contractures (Schwarze et al., 2013a; Caparros-Martin et al., 2013). A specific homozygous $F K B P 10$ deletion of a conserved tyrosine residue (c.877_879del; p(Tyr293del); LOVD FKBP10_00017) was reported uniquely in a Yup'ik Inuit population to cause Kuskokwim syndrome (KS), an arthrogryposis syndrome with less pronounced fracturing that expands the phenotypic range of FKBP65-related skeletal dysplasia. Hitherto, it is unknown if there is a specific genotype-phenotype correlation for KS (Barnes et al., 2013; Petajan et al., 1969).

Here we present an extended consanguineous Palestinian family with a homozygous FKBP10 mutation in which the three above mentioned syndromes, OI, BS1 and KS, are represented.

\subsection{Patient data}

Previously, we reported on eight patients of a consanguineous Palestinian family harboring the novel homozygous splice site variant c. $391+4 \mathrm{~A}>\mathrm{T}$ in the FKBP10 gene (OI variant database (https://oi. gene.le.ac.uk); LOVD DB ID FKBP10_00031) (Essawi et al., 2018; Dalgleish, 1997, 1998). All eight patients were diagnosed with Bruck syndrome based on recurrence of long bone fractures and congenital contractures. We now re-evaluated the disease spectrum in this family in detail and obtained thirteen additional blood samples (IV: 1-8 \& V: $1,10,16,17$ \&19) for variant analysis. Informed consent from the participant and/or the legal guardian was obtained, and the study was approved by the ethics committee of the Ghent University Hospital and was performed in compliance with the Declaration of Helsinki.

\section{Methods}

\subsection{Cell culture and isolation of DNA and RNA}

Genomic DNA was isolated and purified from whole EDTA-blood by Qiagen DNeasy Kit using standard protocols (Qiagen, Frankfurt, Germany). A skin biopsy was obtained from the proband (V:6). Cells were cultured in Dulbecco's modified Eagle's medium (DMEM) supplemented with $10 \%$ fetal bovine serum (FBS), $10 \mathrm{IU} / \mathrm{ml}$ penicillin, 100 $\mu \mathrm{g} / \mathrm{ml}$ kanamycin, $25 \mu \mathrm{g} / \mathrm{mL}$ fungizone, and $1 \%$ non-essential amino acid (NEAA) Solution 100X (all purchased from Invitrogen ${ }^{\mathrm{Tm}}$, Thermo Fisher Scientific, Inc., Waltham, MA, USA), and incubated at $37{ }^{\circ} \mathrm{C}$ with $5 \%$ CO2. RNA was isolated with the RNeasy mini kit (Qiagen, Frankfurt, Germany). Subsequently, cDNA was synthesized using the MMLV cDNA synthesis kit according to the manufacturer's instructions (Qiagen, Frankfurt, Germany).

\subsection{Sequencing and expression analysis}

The splice site variant and exon skipping were confirmed by bidirectional Sanger Sequencing using an ABI3730XL sequencer (Applied Biosystems, Inc., Foster City, CA) at gDNA and cDNA level, respectively. Nomenclature is based on the HGMD guidelines and refers to NCBI reference sequence NM_021939.3 for FKBP1O and NP_068758.3 for FKBP65. Gene expression was investigated by reverse-transcription quantitative PCR (RT-qPCR) (primers and conditions available upon request). Data was analyzed by qBase Plus (Biogazelle, Ghent, Belgium) using the reference genes HPRT1, YWHAZ, and RPL13A for normalization.

\subsection{Western blotting and protein analysis}

Patient and control fibroblasts' cell lysate were extracted using NP40 lysis buffer (Thermo Fisher Scientific, Merelbeke, Belgium), according to the manufacturer's protocol. Western blotting was performed using electrophoresis system with NuPage 4-12\% Bis-Tris $1.5 \mathrm{~mm} \times 10$ wells mini gels and transferred to a Nitrocellulose membrane (Life Technologies Europe, Merelbeke, Belgium). Precision Plus Protein All Blue standard was used for monitoring electrophoretic separation and molecular weight sizing (Bio-Rad Laboratories, Temse, Belgium). The membrane was blocked with 3\% ECL blocking agent (Fisher Scientific, Brussels, Belgium). Afterwards, the membrane was incubated overnight with 1:500 Anti-FKBP10 (HPA051171) primary antibody (SigmaAldrich, Overijse, Belgium). Subsequently, the membrane was washed and incubated with 1:2500 anti-rabbit secondary antibody (Abcam, Cambridge, UK). $\alpha$-Tubulin (1:1000) was used as a protein loading control (Sigma-Aldrich, Overijse, Belgium).

\subsection{HP and LP cross-linking analysis}

Urine samples obtained from five patients (V:5, V:9, V:12, V:13, and $\mathrm{V}: 19$ ) and three non-affected family members (IV:8, V:15, V:17) were hydrolyzed in $6 \mathrm{~N} \mathrm{HCl}$, dried and the material re-dissolved in $10 \%$ acetic acid. The soluble material was loaded on a Bio-Gel P-2 column $(1.5 \mathrm{~cm} \times 30 \mathrm{~cm}$, (Bio-Rad Laboratories, CA, United States) and then eluted with $10 \%$ acetic acid. The fractions that contained pyridinolines were pooled for quantitative analysis by C18 reverse-phase HPLC (Eyre, 1987).

\section{Results}

\subsection{Molecular analysis and phenotypic description}

The pedigree of the family is presented in Fig. 1. Molecular analysis revealed that all patients' parents (IV: 1-8) and two further family members (V:10 \&17) were heterozygous carriers of the splice site variant c. $391+4 \mathrm{~A}>\mathrm{T}$ in the FKBP10 gene (OI variant database (https:// oi.gene.le.ac.uk); LOVD DB ID FKBP10_00031). Two other family members did not carry the variant. None of these individuals presented with a skeletal phenotype. Patient V:19, a six years old boy, was diagnosed with arthrogryposis and was found to harbor the same homozygous splice site variant. He only showed congenital contractures without any fractures. In addition, one OI patient (V: 11), presenting with the most severe phenotype, according to the family, died at the age 23 years due to the complications of progressive kyphoscoliosis causing respiratory deficiency and pain (no DNA available). Another affected family member (V: 8) died at the age of 32 years suffering from similar symptoms with shortness of breath and chest pain. Overall, patient ages ranged from 6 to 46 years. The main clinical features reported are blue sclerae (10/10) (becoming less obvious with increasing age), progressive kyphoscoliosis $(7 / 10)$, club feet $(6 / 10)$, dentinogenesis imperfecta (3/10) and inguinal hernia (2/10). None of the patients had hearing loss (Table 1). All ten patients were classified according to severity and main presenting features, as indicated in Tables 2 and 3. The first group includes patients with severe OI ( $>100$ fractures) and no or very mild contractures, progressive kyphoscoliosis and lower

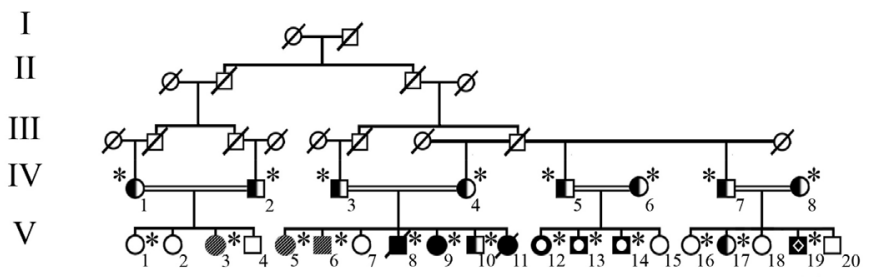

Fig. 1. Pedigrees of the family that participated in this study. Asterisk (*) indicates family members from whom DNA was obtained. Segregation analysis revealed that ten family members designated with half shaded shapes were heterozygous carriers and two were non-carriers. Whereas nine affected individuals of whom DNA was available harbor the homozygous splice site variant. Group 1 patients denoted by black shapes; Group 2 patients denoted by stripped shapes; Group 3 patients denoted by black shapes labeled with an internal white circle; and finally Group 4 patient denoted by a black square labeled with an internal white rhomboid. See Table 2 for the phenotype description of the different groups. 
Table 1

Clinical data of the patients harboring the homozygous splice site variant c.391 $+4 \mathrm{~A}>\mathrm{T}$. Group 1 includes patients with severe OI and no or very mild contractures; Group 2 includes patients with moderate OI and mild to moderate arthrogryposis; Group 3 includes patients with mild OI and moderate to severe arthrogryposis; Group 4 includes the patient with severe arthrogryposis and no fractures. See Fig. 4.

$\dagger^{*}$ Died at the age of 32 and 23 respectively.

$\$$ Patient ID corresponds to the identifiers found in Fig. 1: Family Pedigree.

$\S+,++,+++$ or ++++ indicates the severity.

\begin{tabular}{|c|c|c|c|c|c|c|c|c|c|c|c|c|}
\hline ID & Sex & Age & $\begin{array}{l}\text { Recurring } \\
\text { fractures }\end{array}$ & $\begin{array}{l}\text { Blue } \\
\text { Sclera }\end{array}$ & Club Feet & $\begin{array}{l}\text { Pectus } \\
\text { deformity }\end{array}$ & Kyphoscoliosis & Contractures & Hernia & $\begin{array}{l}\text { Dentinogenesis } \\
\text { Imperfecta }\end{array}$ & $\begin{array}{l}\text { Wheel-chair } \\
\text { bound }\end{array}$ & $\begin{array}{l}\text { Phenotypic } \\
\text { group }\end{array}$ \\
\hline $\mathrm{V}: 3$ & $\mathrm{~F}$ & 12 & $>30$ & + & + & + & + & ++ & - & - & + & 2 \\
\hline V:5 & $\mathrm{F}$ & 46 & $>50$ & + & + & + & - & ++ & + & + & + & 2 \\
\hline $\mathrm{V}: 6$ & M & 44 & $>50$ & + & + & + & - & ++ & + & + & + & 2 \\
\hline $\mathrm{V}: 8$ & M & $32 *$ & $>100$ & + & - & + & + & + & - & - & + & 1 \\
\hline V:9 & $\mathrm{F}$ & 34 & $>100$ & + & - & + & + & + & - & - & + & 1 \\
\hline V:11 & $\mathrm{F}$ & $23^{*}$ & $>100$ & + & - & + & + & - & - & - & + & 1 \\
\hline $\mathrm{V}: 12$ & $\mathrm{~F}$ & 18 & 20 & + & + & + & + & +++ & - & - & + & 3 \\
\hline V:13 & M & 17 & 17 & + & + & + & + & +++ & - & + & + & 3 \\
\hline $\mathrm{V}: 14$ & M & 9 & 9 & + & + & + & + & +++ & - & - & + & 3 \\
\hline V:19 & M & 6 & 0 & + & - & - & - & ++++ & - & - & - & 4 \\
\hline
\end{tabular}

limbs hypotonia. The second group presented with moderate OI $(>50$ fractures) with mild to moderate arthrogryposis, no or mild kyphoscoliosis, hypotonia of the lower limbs, inguinal hernias and club feet. The third group showed mild OI ( $<20$ fractures) with moderate to severe arthrogryposis, mild to moderate kyphoscoliosis, upper and lower limb hypotonia, and club feet. The fourth group contains patients with severe arthrogryposis without any fractures, and hypotonia of the upper and lower limbs. An inverse relationship between the severity of the OI and the contractures is observed (Fig. 4).

\subsection{Functional analysis}

We investigated the effect of the c. $391+4 \mathrm{~A}>\mathrm{T}$ variant at the cDNA level, revealing a 146 bp out-of-frame deletion of exon 2, resulting in a deletion of 48 amino acids and a premature stop codon. RTqPCR of FKBP10 expression showed a 10-fold down regulation in the proband V:6 compared to an age and sex matched control. Loss of expression was confirmed by the absence of FKBP65 on Western blotting in the proband's fibroblasts. All results are illustrated in Fig. 2.

\subsection{HP and LP cross-linking analysis}

Hydroxylysyl pyridinoline (HP) and lysyl pyridinoline (LP) crosslinking was measured in urine samples of five patients and three nonaffected family member. The HP to LP ratios obtained are shown in Sup. Table 2. Control samples (IV:8, V:15 and V:17) show ratios within the normal range. Patients HP/LP are within normal range for the two older patients V:5 and V:9, but higher than normal for the younger patients $\mathrm{V}: 12, \mathrm{~V}: 13$ and V:19. The latter observation is in concordance with previous findings in patients with FKBP10 mutations and appears to account for HP derived from calcified cartilage type II collagen and not
Table 3

Variant effect versus severity of the phenotype. A chi-square test to verify if frameshift variants in FKBP10 are more likely to cause OI rather than BS is not significant (p-value of 0.310494; $\mathrm{p}<0.5$ ).

\begin{tabular}{lllllll}
\hline Effect & Total \# & OI & BS & KS & OI + BS & OI + BS + KS \\
Frameshift & $21 / 45$ & 6 & 12 & & 3 & \\
Nonsense & $10 / 45$ & 6 & 4 & & & \\
Missense & $5 / 45$ & 2 & 2 & & 1 & 1 \\
Splice-site & $5 / 45$ & 4 & & & & \\
In-Frame & $3 / 45$ & 1 & 1 & 1 & & \\
Other & $1 / 45$ & 1 & & & & \\
\hline
\end{tabular}

bone type I collagen (Schwarze et al., 2013a). Hence, the increased ratios in younger patients may relate to active growth where type II collagen is continuously broken down from the growth plate.

\section{Discussion}

We report on an extended Palestinian consanguineous family with 10 affected family members presenting a phenotypic spectrum ranging from severe OI to isolated arthrogryposis. To date 45 disease-causing variants are reported in the FKBP10 gene (Fig. 3 and Sup. Table 3), originating from different populations, making FKBP10 the second most prevalent recessive OI gene after P3H1 (OMIM 610915) according to the OI variant database (Dalgleish, 1997, 1998). Fig. 3 illustrates that FKBP10 pathogenic variants are distributed over the entire gene. Most pathogenic variants are frameshift variants $(21 / 45)$, followed by nonsense $(10 / 45)$ missense $(5 / 45)$, splice site $(5 / 5)$ and in-frame $(3 / 45)$ variants. There are no clear genotype-phenotype correlations with any of three disease presentations (Table 3). Four variants (4/45) cause both $\mathrm{BS}$ and OI, and one (1/45) in-frame deletion variant cause KS

Table 2

Phenotype description of the patients harboring the homozygous splice site variant c.391 +4A $>$ T. Comparison between the different patient groups according to the phenotypes presented.

\begin{tabular}{|c|c|c|c|c|}
\hline & $\begin{array}{l}\text { Group1 } \\
\text { V: } 8,9 \& 11\end{array}$ & $\begin{array}{l}\text { Group } 2 \\
\text { V: } 3,5 \& 6\end{array}$ & $\begin{array}{l}\text { Group } 3 \\
\text { V: } 12,13 \& 14\end{array}$ & $\begin{array}{l}\text { Group } 4 \\
\text { V: } 19\end{array}$ \\
\hline Phenotype & Severe OI/No or mild Ar & Moderate OI/Mild-Moderate Ar & Mild OI/Moderate-Severe Ar & Arthyrogryposis (Ar) \\
\hline Fractures & $>100$ & $>50$ & $<25$ & No \\
\hline Contractures & No or Hardly detectable & Mild & Moderate & Severe \\
\hline Upper limbs hypotonia & No & No & Yes & Yes \\
\hline Lower limbs hypotonia & Yes & Yes & Yes & Yes \\
\hline Kyphoscoliosis & Severe & No or mild & Mild - Moderate & No \\
\hline Dentinogenesis Imperfecta & No & Yes & Yes & No \\
\hline Blue sclaere & Diminishes with age & Diminishes with age & Diminishes with age & Diminishes with age \\
\hline Inguinal Hernia & No & Yes & No & No \\
\hline Club foot & No & Yes & Yes & No \\
\hline
\end{tabular}


A

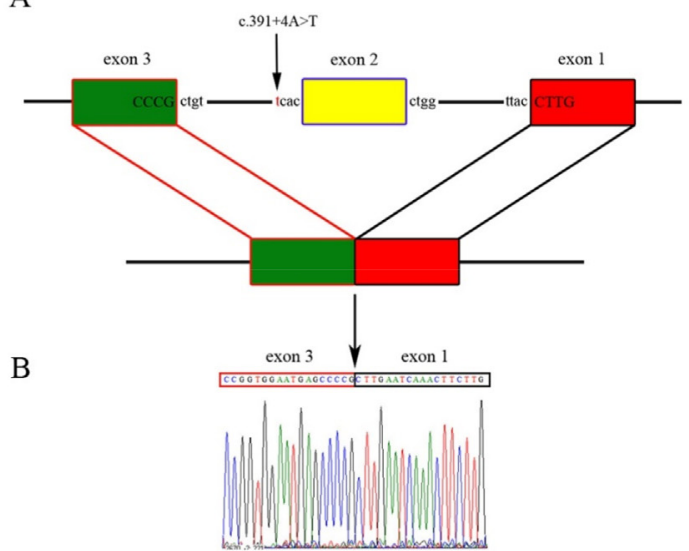

C

\section{FKBP10 Expression}
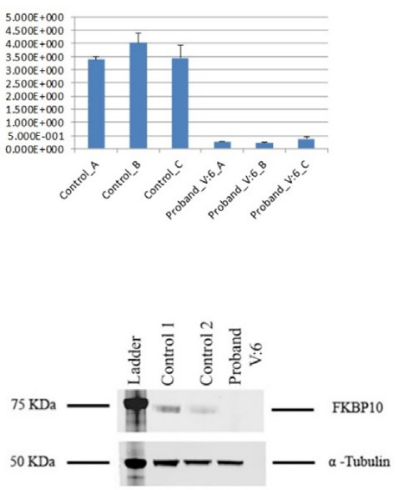

Fig. 2. Functional Data. A: Schematic representation of the aberrant splicing process in FKBP10. The Boxes denote the exons, and the solid lines indicate the introns. B: c.DNA sequencing of the proband V: 6 harboring the homozygous splice site variant c. $391+4 \mathrm{~A}>\mathrm{T}$ revealed skipping of exon 2 of the FKBP1O gene, predicted to result in an out-of-frame deletion of 48 amino acids (146 bp). C: FKBP10 gene expression indicating 10 fold down regulation in the proband V:6 sample compared to a control sample. D: Western blotting assay revealed the absence of FKBP65 in the probands fibroblasts; Lane 1 indicates all blue ladder, lane 2 indicates an immortalized BJ5ta fibroblast cell line derived by transfecting the BJ foreskin fibroblast cell line with the pGRN145 hTERT-expressing plasmid and obtained from a neonatal male, lane 3 indicates an age and sex matched control fibroblasts and lane 4 indicates the proband V:6 fibroblasts. (For interpretation of the references to colour in this figure legend, the reader is referred to the Web version of this article.)
(Table 3 \& Sup. Fig. 1). The most frequent FKBP10 variant c.831dupC p. (Gly278Argfs $* 95$ ), was reported both for OI and BS, but not for KS in several population groups worldwide (Alanay et al., 2010; Shaheen et al., 2011; Kelley et al., 2011; Schwarze et al., 2013a; Caparros-Martin et al., 2013, 2017; Vorster et al., 2017; Mrosk et al., 2018; Xu et al., 2017; Umair et al., 2016; Alabdullatif et al., 2017).

Several reports describe inter- and intra-familial variability encompassing both BS and OI related phenotypes (Alanay et al., 2010; Shaheen et al., 2010, 2011; Kelley et al., 2011; Schwarze et al., 2013a; Caparros-Martin et al., 2017). However, in all OI and BS families reported with $F K B P 10$ mutations, there are no patients without a fracture and only contractures, except for the Kuskokwim cohort. This suggests that the KS phenotype is peculiar to the specific FKBP10 variant that result in an in-frame deletion of the conserved p.Tyr293 in FKBP65 (Barnes et al., 2013). It was suggested that OI and BS phenotypes might result from absent FKBP65 protein and undetectable hydroxylation of type I collagen telopeptide lysines, whereas the in-frame deletion mutation causing KS destabilized the FKBP65 protein. A residual amount that accounted for an observed 2-10\% hydroxylation of the telopeptide lysines was observed (Barnes et al., 2013). In our study, proband V:6 (diagnosed with BS) had a 10-fold reduction in FKBP10 gene expression

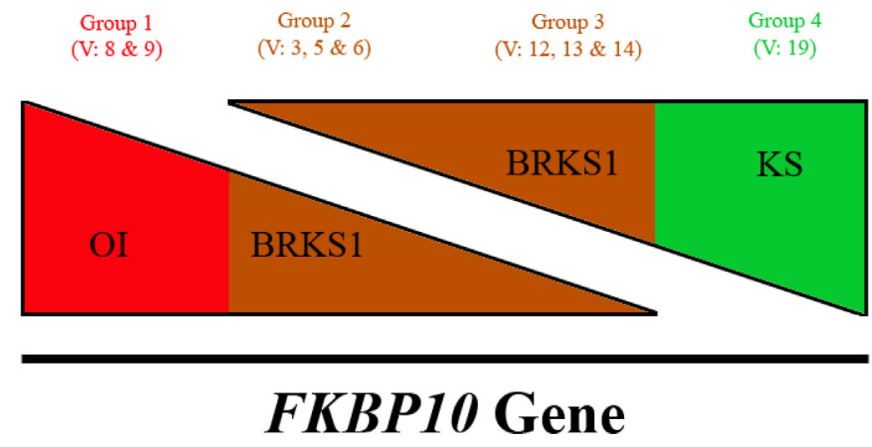

Fig. 4. Phenotypic heterogeneity in FKBP1O family. Schematic diagram of the phenotype of $F K B P 10$ variants illustrating the inverse relationship between the severity of the OI and the contractures.

This diagram was derived and adapted from a publication of Barnes et al., 2013)

compared to controls, but no residual FKBP65 protein (Fig. 2).

FKBP10 deficiency results in reduced hydroxylation of telopeptide lysine of type I collagen in bone or cultured fibroblast samples (Barnes et al., 2012, 2013; Schwarze et al., 2013a; Duran et al., 2017; Bank

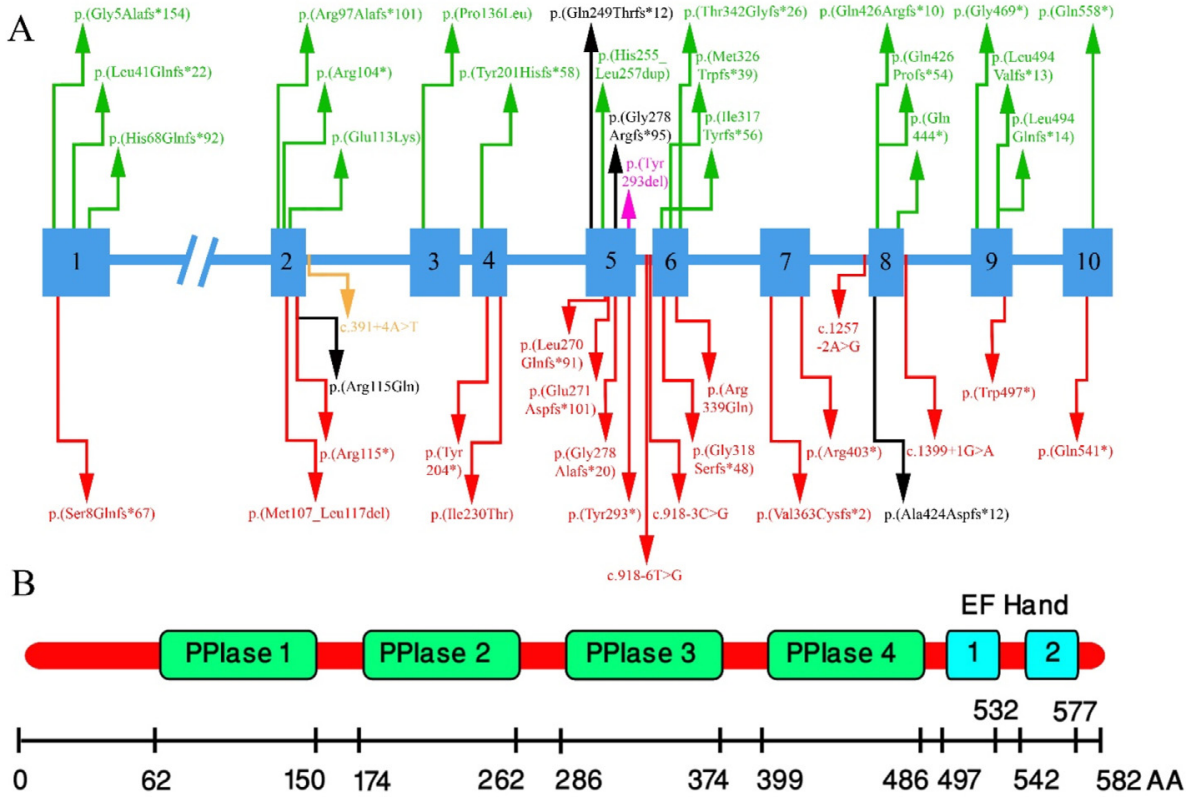

Fig. 3. FKBP10 variants. A-Pathogenic variants associated with the BS are indicated with green arrows and shown in the upper side of the figure, pathogenic variants associated with OI are indicated in red arrows and shown in the lower part. Black arrows indicates pathogenic variants causing both BS and OI. The orange arrow indicates the pathogenic variant identified in this study causing BS, OI and KS and finally the pink arrow indicates the in frame deletion causing KS. Transcript sequence NM_021939.3 of the FKBP10 gene consists of 10 coding exons. Most of the OI causing variants were found in exon 5 and 6. BFKBP65 protein NP_068758.3 consists of six domains: (i) PPIase FKBP-type 1 (residues 62-150), (ii) PPIase FKBP-type 2 (residues 174-262), (iii) PPIase FKBP-type 3 (residues 286-374), (iv) PPIase FKBPtype 4 (residues 399-486), (v) EF-hand 1 (residues 497-532) and (vi) EF-hand 2 (residues 542-577). Detailed information about all disease causing variants in the FKBP10 gene are available in Sup. Table 3. * The genomic deletion variant g.39974881_39980318del (hg19) is not indicated on the graph. . (For interpretation of the references to colour in this figure legend, the reader is referred to the Web version of this article.) 
et al., 1999). Hydroxylated telopeptide lysine varied from less than $1 \%$ (controls 60\%) in patients' fibroblasts with null mutations and moderate-severe OI (Barnes et al., 2012) to 4-6\% hydroxylation (controls $75 \%$ ) in bone samples of patients with a missense mutation and BS (Bank et al., 1999; Schwarze et al., 2013b). Furthermore, Schwarze et al. examined the hydroxylation of telopeptide lysine in a bone sample of a BS patient with a null mutation revealing about $10 \%$ hydroxylation of telopeptide lysine in comparison to a control. In KS patients due to the p.Tyr293del variant telopeptide lysyl hydroxylation varied between 2 and 10\% (controls 60\%) (Barnes et al., 2013). Unfortunately, because of the different tissues (bone vs fibroblasts) and different effect of the mutations (null vs missense) analyzed, no conclusive results can be obtained concerning the relation between the amount of telopeptide lysine hydroxylation and the severity of the phenotype. Seemingly contradictory, HP/LP crosslinking is higher in patient versus control urine samples. This can be explained by a significant contribution to urine HP by growth plate cartilage type II collagen breakdown for the patient samples. We know that telopeptide hydroxylation of type II collagen is unaffected by FKBP65 deficiency (Schwarze et al., 2013b). Higher urine HP/LP values were inversely correlated with OI phenotypes, a trend we could confirm in our patients (Sup. Table 2).

Our report highlights the wide clinical spectrum in our family indicating that the three FKBP10-related disorders, OI, BS and KS, can be caused by a same mutation.

Besides the inverse relationship between the severity of OI and the contractures (Fig. 4), other manifestations including inguinal hernia, club feet and dentinogenesis imperfecta did not seem to correlate with the primary clinical diagnosis of either OI (Group 1 patients (V: 8, 9 \& 11)) or arthrogryposis (Group 4 (V: 19) patient) (Table 2).

In conclusion, our data confirm the wide clinical spectrum and intra-familial variability in FKBP1O related disease, and suggests that it could contribute to the mutational spectrum of isolated arthrogryposes.

\section{CRediT authorship contribution statement}

Osama H. Essawi: Investigation, Validation, Resources, Writing original draft, Visualization. Piyanoot Tapaneeyaphan: Investigation, Validation. Sofie Symoens: Writing - review \& editing. Charlotte Gistelinck C: Investigation, Validation. Fransiska Malfait: Writing review \& editing. David R. Eyre: Writing - review \& editing, Supervision. Tamer Essawi: Supervision. Bert Callewaert: Writing review \& editing. Paul J. Coucke: Supervision, Writing - review \& editing, Visualization, Funding acquisition.

\section{Acknowledgements}

We thank all the patients and their families who supported our research efforts. We are grateful to Ahmad Thwaeb and Omar Mohammad for blood and urine sampling.

\section{Appendix A. Supplementary data}

Supplementary data to this article can be found online at https:// doi.org/10.1016/j.ejmg.2020.103980.

\section{Funding sources}

F. M. and B. C. are fellows of the Fund for Scientific Research
(FWO). O. E. has a BOF Research Fellowship from the Ghent University. Contract grants include FWO grant number G.0171.05 and Methusalem grant number 08/01M01108 to A. D. P.

\section{References}

Alabdullatif, M.A., et al., 2017. Chromosomal microarray in a highly consanguineous population: diagnostic yield, utility of regions of homozygosity, and novel mutations. Clin. Genet. 91 (4), 616-622.

Alanay, Y., et al., 2010. Mutations in the gene encoding the RER protein FKBP65 cause autosomal-recessive osteogenesis imperfecta. Am. J. Hum. Genet. 86 (4), 551-559.

Bank, R.A., et al., 1999. Defective collagen crosslinking in bone, but not in ligament or cartilage, in Bruck syndrome: indications for a bone-specific telopeptide lysyl hydroxylase on chromosome 17. Proc. Natl. Acad. Sci. U.S.A. 96 (3), 1054-1058.

Barnes, A.M., et al., 2012. Absence of FKBP10 in recessive type XI osteogenesis imperfecta leads to diminished collagen cross-linking and reduced collagen deposition in extracellular matrix. Hum. Mutat. 33 (11), 1589-1598.

Barnes, A.M., et al., 2013. Kuskokwim syndrome, a recessive congenital contracture disorder, extends the phenotype of FKBP10 mutations. Hum. Mutat. 34 (9), 1279-1288.

Bonafe, L., et al., 2015. Nosology and classification of genetic skeletal disorders: 2015 revision. Am. J. Med. Genet. 167A (12), 2869-2892.

Caparros-Martin, J.A., et al., 2013. Clinical and molecular analysis in families with autosomal recessive osteogenesis imperfecta identifies mutations in five genes and suggests genotype-phenotype correlations. Am. J. Med. Genet. 161A (6), 1354-1369.

Caparros-Martin, J.A., et al., 2017. Molecular spectrum and differential diagnosis in patients referred with sporadic or autosomal recessive osteogenesis imperfecta. Mol. Genet. Genom. Med. 5 (1), 28-39.

Dalgleish, R., 1997. The human type I collagen mutation database. Nucleic Acids Res. 25 (1), 181-187.

Dalgleish, R., 1998. The human collagen mutation database 1998. Nucleic Acids Res. 26 (1), 253-255.

Duran, I., et al., 2017. A chaperone complex formed by HSP47, FKBP65, and BiP modulates telopeptide lysyl hydroxylation of type I procollagen. J. Bone Miner. Res. : Off. J. Am. Soc. Bone Min. Res. 32 (6), 1309-1319.

Essawi, O., et al., 2018. Genetic analysis of osteogenesis imperfecta in the Palestinian population: molecular screening of 49 affected families. Mol. Genet. Genom. Med. 6 (1), 15-26.

Eyre, D., 1987. Collagen cross-linking amino acids. Methods Enzymol. 144, 115-139.

Forlino, A., Marini, J.C., 2016. Osteogenesis imperfecta. Lancet 387 (10028), 1657-1671.

Ishikawa, Y., et al., 2008. The rough endoplasmic reticulum-resident FK506-binding protein FKBP65 is a molecular chaperone that interacts with collagens. J. Biol. Chem. 283 (46), 31584-31590.

Kelley, B.P., et al., 2011. Mutations in FKBP10 cause recessive osteogenesis imperfecta and Bruck syndrome. J. Bone Miner. Res. : Off. J. Am. Soc. Bone Min. Res. 26 (3), 666-672.

Mrosk, J., et al., 2018. Diagnostic strategies and genotype-phenotype correlation in a large Indian cohort of osteogenesis imperfecta. Bone 110, 368-377.

Petajan, J.H., et al., 1969. Arthrogryposis syndrome (Kuskokwim disease) in the eskimo. J. Am. Med. Assoc. 209 (10), 1481-1486.

Puig-Hervas, M.T., et al., 2012. Mutations in PLOD2 cause autosomal-recessive connective tissue disorders within the Bruck syndrome-osteogenesis imperfecta phenotypic spectrum. Hum. Mutat. 33 (10), 1444-1449.

Schwarze, U., et al., 2013a. Mutations in FKBP10, which result in Bruck syndrome and recessive forms of osteogenesis imperfecta, inhibit the hydroxylation of telopeptide lysines in bone collagen. Hum. Mol. Genet. 22 (1), 1-17.

Schwarze, U., et al., 2013b. Mutations in FKBP10, which result in Bruck syndrome and recessive forms of osteogenesis imperfecta, inhibit the hydroxylation of telopeptide lysines in bone collagen. Hum. Mol. Genet. 22 (1), 1-17.

Shaheen, R., et al., 2010. FKBP10 and Bruck syndrome: phenotypic heterogeneity or call for reclassification? Am. J. Hum. Genet. 87 (2), 306-307 author reply 308.

Shaheen, R., et al., 2011. Mutations in FKBP10 cause both Bruck syndrome and isolated osteogenesis imperfecta in humans. Am. J. Med. Genet. 155A (6), 1448-1452.

Sillence, D.O., Senn, A., Danks, D.M., 1979. Genetic heterogeneity in osteogenesis imperfecta. J. Med. Genet. 16 (2), 101-116.

Umair, M., et al., 2016. Homozygous sequence variants in the FKBP10 gene underlie osteogenesis imperfecta in consanguineous families. J. Hum. Genet. 61 (3), 207-213.

Vorster, A., et al., 2017. Osteogenesis imperfecta type 3 in South Africa: causative mutations in FKBP10. South African medical journal = Suid-Afrikaanse tydskrif vir geneeskunde 107 (5), 457-462.

Xu, X.J., et al., 2017. Novel mutations in FKBP10 in Chinese patients with osteogenesis imperfecta and their treatment with zoledronic acid. J. Hum. Genet. 62 (2), 205-211. 